\title{
Vulvar Craurosis due to Severe Genitourinary Syndrome of Menopause Complicated by Lichen Sclerosus: Two Case Reports and Literature Review
}

\author{
Marta Pérez Febles ${ }^{1}$, Victoria Pascual Escudero ${ }^{1}$, Sonia De Miguel Manso ${ }^{1,2}$, María López Pais ${ }^{1}$, Dakota Viruega Cuaresma ${ }^{1}$, Paula Suárez \\ Mansilla ${ }^{1,2}$ \\ ${ }^{1}$ Servicio de Obstetricia y Ginecología, Hospital Clínico Universitario de Valladolid, Gerencia Regional de Salud de Castilla y León (SACYL), Spain. \\ ${ }^{2}$ Departamento de Pediatría e Inmunología, Obstetricia y Ginecología, Nutrición y Bromatología, Psiquiatría e Historia de la Ciencia, Facultad de \\ Medicina, Universidad de Valladolid, Spain.
}

Corresponding Author: Marta Pérez Febles, Avenida Ramón y Cajal 3, 47005 Valladolid, España.

Received date: September 29, 2021; Accepted date: October 19, 2021; Published date: November 19, 2021

Citation: Marta P. Febles, Victoria P. Escudero, Sonia D.M. Manso, María L. Pais, Dakota V. Cuaresma, Paula S. Mansilla (2021) Vulvar Craurosis due to Severe Genitourinary Syndrome of Menopause Complicated by Lichen Sclerosus: Two Case Reports and Literature Review. J.Obstetrics Gynecology and Reproductive Sciences 5(9); DOI: 10.31579/2578-8965/100

Copyright: (C) 2021, Marta Pérez Febles, This is an open access article distributed under the Creative Commons Attribution License, which permits unrestricted use, distribution, and reproduction in any medium, provided the original work is properly cited.

\begin{abstract}
Background: Vulvovaginal atrophy affects approximately $45 \%$ of middle-aged women, as a result of decreased estrogen circulating levels and is often associated with vulvovaginal discomfort in menopausal patients. The spectrum of adverse consequences makes long-term treatment essential, not only for symptom relief, but also to avoid further complications.

These symptoms can even be worsened if other vulvar conditions coexist, such as inflammatory dermatoses.

Clinical presentation: We present two cases of patients with severe vulvar atrophy and craurosis, who required vulvo-vaginal opening associated with medical treatment. In both patients, we diagnosed severe genitourinary syndrome of menopause (GSM) complicated by vulvar lichen sclerosus, a very rare clinical situation. In case 1, the symptoms had progressed despite local estrogen treatment for vulvovaginal atrophy, and lichen wasn't considered in previous check-ups. In contrast, patient $\mathrm{n}^{\circ} 2$ presented in the ER with impossibility to urinate and was then diagnosed and treated for underlying causes.

Conclusions: GSM treatment should be a priority. The absence of improvement or progression of genital atrophy should alert the specialist to the coexistence of other vulvar pathologies.

Keywords: vulvar craurosis, SGM; menopause; lichen sclerosus;
\end{abstract}

\section{Abbreviations:}

GSM: Genitourinary syndrome of menopause.

HT: hormone therapy

ER: Emergency room.

LS: lichen sclerosus.

\section{Introduction}

Genitourinary syndrome of menopause (GSM) is defined as the set of symptoms and signs caused by decrease in circulating estrogens. Its evolution is chronic and progressive. This syndrome may include genital symptoms such as dryness, burning or irritation; sexual symptoms due to lack of lubrication or pain and urinary symptoms and recurrent urinary infections.

These clinical modifications are also associated with the loss of sexual activity and / or sexual dysfunctions, causing a negative impact on quality of life in up to half of postmenopausal patients. Therefore, it is one of the most common reasons for consultation. Currently, this 
condition continues to be underdiagnosed and undertreated, due to patient's shyness and failure in doctor-patient communication.

These symptoms can even be worsened if other vulvar conditions coexist, such as inflammatory dermatoses.

The objective of this manuscript is to present two cases with severe vulvar atrophy worsened by lichen sclerosus coexistence. As a consequence of suboptimal treatment, it led to an uncommon but severe complication, known as vulvar craurosis who required finally vulvovaginal opening.

\section{Clinical Cases Case 1}

A 61-year-old woman with no relevant clinical history, with menopause at 57 years and two vaginal deliveries, who consulted for long-term dyspareunia, urinary difficulty and genital itching. Local treatments (first hydration then hormonal) were prescribed previously by her general doctor without improvement. She has required urethral dilations performed by an urologist, without further diagnostic process.

She was referred for assessment in our department. During examination, vaginal cleisis was observed, visualizing a septum from anterior to posterior vaginal wall with a thickness of $3 \mathrm{~cm}$, trapping an estrogen ring that she inserted 6 months ago, after medical prescription. The atrophic cervix was retracted in the vaginal fundus. Furthermore, hypo pigmented hyperkeratotic lesions were described on both labia major.

We decided to perform under sedation and local anesthesia, as major outpatient surgery, section of the vaginal septum, with release and opening of the vagina, extraction of the estrogen ring and biopsy of the vulvar skin on the left introitus with dermatological punch $\mathrm{N}^{\circ} 6$. The patient began systemic hormone therapy (HT) with estriol $1 \mathrm{mg} /$ noertisterone acetate $0.5 \mathrm{mg}$ and the use of progressive vaginal dilators, as well as local hydration.

Histological analysis of vulvar skin revealed alterations compatible with lichen sclerosus, including lichenoid interface dermatitis, sclerous thickening of the superficial dermis and ectatic vessels.

The patient attended one month post-surgical check-up, presenting little clinical improvement with persistence of intense genital itching. Vaginal exam was still impossible due to pain, but we noticed persistence of vulvar lesions with with erythema and smooth whitish

\section{Case 2}

61-year-old woman, hypertensive, with menopause at age 50 and a vaginal birth. She consulted in the ER referring hypogastric pain that radiates to lumbar region accompanied by nausea and constipation, initiated 6 months ago but that has increased in the last 30 days.

She was treated, unsuccessfully, for recurrent urinary tract infections. Examination revealed a complete vulvar craurosis with a millimetric opening in the upper third, leaking urine drops. Vaginal introitus is not identified and there is general hypopigmentation of the labia majora with hyperkeratotic-like lesions on both lips, perianal region, and central fusion line that occludes urethral meatus, introitus, and clitoris (Figure 2). Similar lesions are seen in the crural grooves and in the right inguinal region, a violaceous macula with a reticular appearance. plaques. We prescribed then treatment with high-potency topical corticosteroids (methylprednisolone aceponate), continuing with oral $\mathrm{HT}$ and the use of vaginal dilators.

At six months check-up, she presented significant clinical improvement. She had tolerated the smallest vaginal dilator. During vaginal exploration, she allowed unidigital examination unpainfully. Vulvar hyperkeratotic lesions had practically disappeared and erythematous lesions linked to atrophy had improved. We added to her treatment in order to reinforce SGM treatment, prasterone combined with HT and we switch to LS maintenance therapy.

After 12 months therapy, she is stable with major improvement of itching but persistence of sexual intercourse deficiencies. She tolerates speculum examination, revealing vaginal cleisis in the upper third that hides cervical surface. Vaginal mucosa shows fewer signs of atrophy and a single whitish plaque is observed in the introitus towards the lower third of the left labia majora (Figure 1). We suspend HT and continue only local treatment with daily vaginal prasterone, and LS maintenance therapy vaginal (daily hydration and weekly corticosteroids treatment).

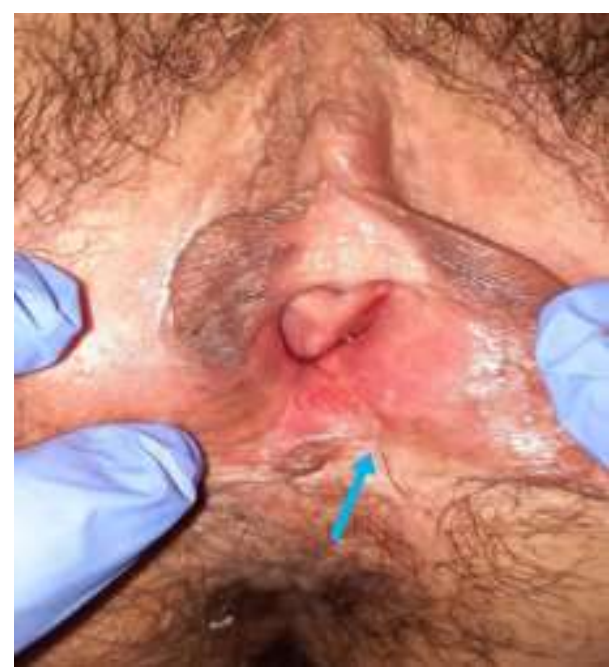

Figure 1

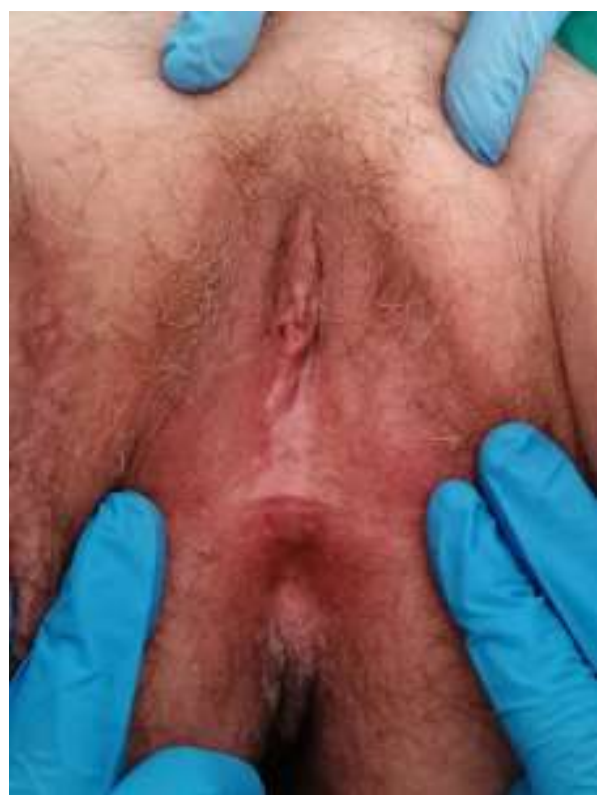


Figure 2

During abdominal ultrasound, we identified distended bladder, compatible with acute urinary retention. Vulvar drainage orifice was enlarged with a Kocher forceps under local anesthetica and antibiotic prophylaxis. A normal-appearing urethral meatus was identified and a permanent bladder catheter was then placed. Vagina was completely closed, precluding to visualize or touch the uterine cervix.

The patient was evaluated ten days after procedure with significant improvement of her clinical situation. The edges of the vulvar opening were healing favorably and urethral meatus was permeable. We removed urinary catheter. We decided to perform a vulvar biopsy with a dermatological punch $\mathrm{N}^{\circ} 5$ under local anesthetica in the lower third of the right lip majora, in order to clarify diagnosis and establish adequate treatment.

At 30 days check-up, patient denied abdominal pain, but complained of slight vulvar itching. A permeable urethral meatus is visualized but vaginal examination remained impossible due to cleisis. One-digit wall separation was attempted with moderate bleeding. Histological analysis revealed sclerosing thickening of the superficial dermis of variable thickness and ectatic vessels, with little inflammatory component, suggestive of lichen sclerosus. She began treatment with high-potency corticosteroids (Clobetasol propionate), vulvar daily hydration, $0.03 \mathrm{mg}$ estriol vaginal ovuli $\mathrm{mg}$ and use of progressively sized vaginal dilators.

After two months of intensive treatment, the patient does not present difficulty in urination or genital itching. She uses external moisturizer every day, local estriol and corticosteroids once a week and continue using vaginal dilators, but she has not attempted sexual intercourse. On

\section{Discussion}

Vulvovaginal atrophy is the result of decreased estrogen levels and is often associated with vulvovaginal discomfort in menopausal patients, as well as recurrent urinary tract infections, among other clinical manifestations (hot flashes, insomnia...)

In 2014, the International Society for the Study of Women's Sexual Health and the North American Menopausal Society introduced the new term genitourinary syndrome of menopause (GSM) [1]. This concept encompasses all atrophic symptoms due to estrogen deficiency, which progressively alters sexual function and quality of life.

Approximately $45 \%$ of middle-aged women experience these symptoms, but the majority go undiagnosed and consequently untreated. The spectrum of adverse consequences makes long-term treatment essential, not only for symptom relief, but also to avoid further complications. Therefore, estrogens are the cornerstone of GSM manifestations [2].

In order to manage local manifestations, first-line treatments are nonhormonal vulvo-vaginal moisturizers. If these do not provide adequate relief of symptoms, progression to local hormonal therapy, either with low-dose vaginal estrogens or with prasterone (an inactive precursor steroid by itself, which converts to estrogens and androgens) is the usual attitude. Both alternatives constitute the most effective treatment for moderate to severe symptoms of vaginal atrophy [3]. They can be administered in various presentations: vaginal gel, ointment, vaginal ovuli, ring... examination: well-shaped labia minora, with slight introitus erythema, urethral meatus without alterations and decreased hypopigmentation in the perianal region (Figure 3). Unidigital vaginal examination is feasible, checking cervix retracted in the vaginal fundus.

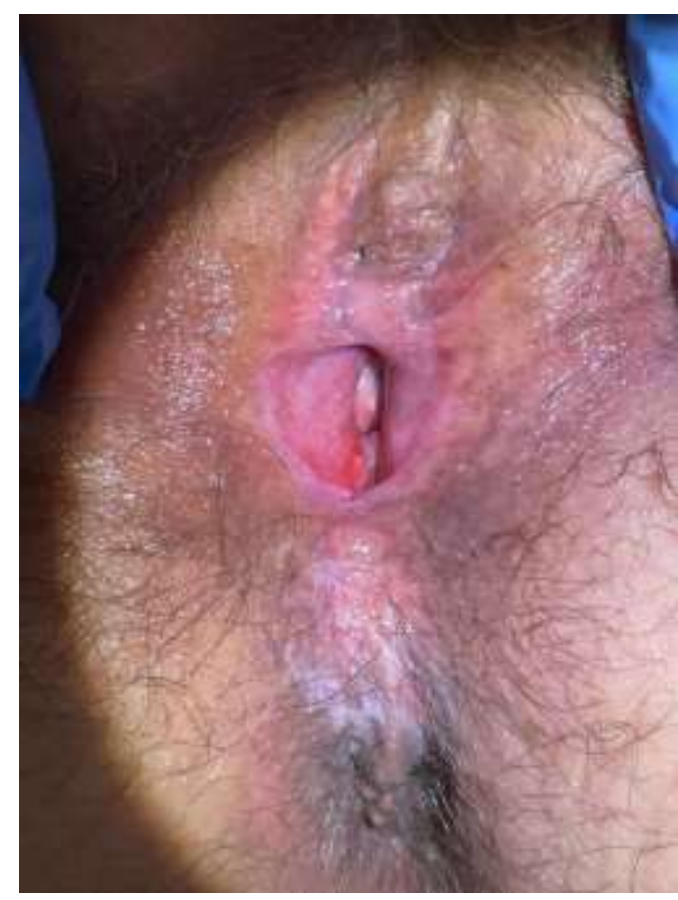

Figure 3

Low-dose vaginal estrogen therapy is recommended in patients with GSM symptoms [4], as indicated by the North American Menopause Society guidelines [5]. Length of treatment necessary to improve symptoms and maintain this improvement can vary widely among patients and should be individualized according to the degree of vaginal atrophy in each patient.

Oral HT is the treatment of choice for vasomotor symptoms of moderate to severe intensity, after failure of non-hormonal measures. In patients with severe GSM and poor response to local treatments, association with oral HT can be considered to achieve greater symptomatic relief [6].

Early treatment start looks for prompt restoration of quality of life, maintain physical and mental state, correct sexual dysfunction and delay the progression of the disease.

Given the proven success of estrogen therapy, the absence of symptom control justifies the evaluation for other etiologies. Likewise, therapy's compliance must be confirmad [7].

GSM's genital atrophy can worsen other vulvar pathologies or mimic associated complications. This coexistence makes treatment challenging. Therefore, recognizing and treating SGM symptoms can improve the management of other vulvovaginal conditions and patient's situation [8].

Lichen sclerosus (LS) is a chronic and progressive inflammatory dermatopathy that usually affects the anogenital region (85-90\%) [9]. It is one of the most frequent causes of gynecological consultation in postmenopausal women, although its prevalence is probably underestimated.

The most frequent manifestation is itching, which is frequently associated with stinging or burning with an evening pattern that can interfere with 
sleep. Later, dyspareunia may appear affecting patient's quality of life in different degrees. As disease progresses, the structural changes secondary to sclerosis and scarring of the vulva are more evident. Vulvar structures are fused and the clitoris can be buried under the fused hood. In the final stages, such as both cases presented, labia majora and minora are sealed leading to a partial to complete occlusion of the introitus (vulvar craurosis) which precludes sexual intercourse and urethral meatusstenosis may be associated, causing difficulty in urination [10].

Therefore, all patients with suspicious symptoms or signs of lichen sclerosus should be referred to a specialist in vulvar pathology and receive treatment.

Diagnosis is mostly clinical. Biopsy is reserved for cases with unclear diagnosis, suspected neoplasic disease, resistance to adequate treatment or atypical extra-genital presentations. This procedure should be done at the interface between normal and affected areas [11].

Differential diagnosis should be made with conditions leading to similar symptoms or common clinical signs. Among the most relevant we can list:

- Vulvar Intraepithelial Neoplasia (VIN): very heterogeneous forms of presentation regarding color, surface and topography.

- Lichen planus: it is characterized by intense itching and alteration of the vulvar morphology, but it usually affects the vagina (and other mucous membranes) producing inflammation and synechiae.

- Lichen simplex chronicus: cutaneous reaction to a chronic aggression and whose main symptom is vulvar itching.

- Severe genital atrophy.

High-potency topical corticosteroids are the mainstay of treatment for lichen sclerosus. If treatment is abandoned, recurrence of symptoms as well as development of sequelae is frequent. For this reason, numerous publications back up maintenance therapy for long periods of time [12].

Either patients with severe GSM or progressive LS may benefit from the use of lubricated dilators of graduated sizes, in order to prevent scarring from causing new stenosis of the introitus or retractions. It is mainly indicated in sexually active patients.

Vulvar self-examination should be recommended to women, to detect changes or alarm signs. This can be difficult in patients with advanced age and comorbidities.

Our two cases presented vaginal cleisis and vulvar fusion due to extreme genital atrophy probably worsened by underlying LS, a very rare clinical situation. In case 1, the GSM symptoms had progressed despite local estrogen treatment, and she had not been diagnosed with lichen in previous check-ups. In contrast, case 2 had not previously used any local therapy and consulted late with an infrequent presentation (acute retention of urine).

Usually, in patients with moderate genital atrophy associated or not with vulvar dermatoses, we find partial fusion of vulvo-vaginal structures, not compromising so significantly their quality of life. Our two exposed cases are particularly severe and lead to uncommon complications, such as urinary or sexual inability. The first patient required urethral dilations and in the second one, the urethral obstruction caused an episode of acute urine retention. None of them was able to have sexual intercourse.

The complexity of treatment in advanced stages and coexistent vulvar pathologies is conditioned by the lack of published data, based on case reports or short series of patients. Some treatment options, such as lasers, have limited use and are practically confined to the field of research [13]. This was the main reason for our attempt in combining treatment strategies (surgical opening + oral HT + local therapies) in the pursue of symptom relief.

\section{Conclusion}

- The treatment of GSM should be a priority since it is related to patient's quality of life and prevention of sequelae.

- The absence of improvement under treatment or progression should alert the specialist in the search for other vulvar diseases, such as inflammatory skin diseases.

- The correct diagnosis of lichen sclerosus and the early initiation of treatment are crucial to prevent the appearance of complications and irreversible scars.

- $\quad$ Since a high percentage of patients with SGM and LS experience a deterioration in quality of life, it is recommended to insist on communication, provision of information and doctor-patient relationship.

\section{Declaration}

\section{Funding:}

'Not applicable'. No funding was obtained for the implementation of this study

\section{Conflicts of interest/Competing interests (include appropriate disclosures):}

'Not applicable'

\section{References:}

1. Kaunitz M and Faubion S. (2021) Menopause The Journal of The North American Menopause Society: health implications and hormonal management Andrew. 28(1).

2. Bachmann G and Santen RJ. (2021) Genitourinary syndrome of menopause (vulvovaginal atrophy): Clinical manifestations and diagnosis. In: Barbieri RL, Chakrabarti A, editors. UpToDate. Waltham, UpToDate;

3. Lethaby A, Ayeleke RO, Roberts H. (2016) Local oestrogen for vaginal atrophy in postmenopausal women. Cochrane Database Syst Rev. 2016(8):CD001500.

4. Bachmann G and Richard J Santen. (2021) Genitourinary syndrome of menopause (vulvovaginal atrophy): Treatment. In: Barbieri RL, Burstein HJ, Chakrabarti A, editors. UpToDate. Waltham.

5. The 2017 hormone therapy position statement of The North American Menopause Society. Menopause. (2018) Nov;25(11):1362-1387.

6. Palacios S, Castelo-Branco C, Cancelo MJ, Vázquez F. (2004) Low-dose, vaginally administered estrogens may enhance local benefits of systemic therapy in the treatment of urogenital atrophy in postmenopausal women on hormone therapy. The European Menopause Journal.

7. Cardozo L, Bachmann G, McCiish D, et al. (1998) Meta-analysis of estrogen therapy in the management of urogenital atrophy in postmenopausal women: second report of the Hormones and Urogenital Therapy Committee. Obstet Gynecol, 92:722.

8. Hum M, Dytoc M. (2017) A Dermatologist's Approach to Genitourinary Syndrome of Menopause. J Cutan Med Surg. 21(5):418-424. doi: 10.1177/1203475417708165. Epub 2017 Apr 28.

9. Cararach M., Castro M., García A., Juliá M., Mascaró J.M, et al. (2016) AEPCC- Guía: Dermatosis inflamatoria de la vulva (liquen escleroso, liquen plano y liquen simple crónico). AEPCC. pp: 1-52. ISBN: 978-84-617-6416-7. 
10. Singh N. and Ghatage P. (2020) Etiology, Clinical Features, and Diagnosis of Vulvar Lichen Sclerosus: A Scoping Review. Obstetrics and Gynecology International. 2020:7480754.

11. Kirtschig G, Becker K, Gunthert A, Jasaitiene D, Cooper S, Chi CC, et al. (2015) Evidence-based (S3) Guideline on (anogenital) Lichen sclerosus. J Eur Acad Dermatol Venereol. 29(10):e1-43.

12. Virgili A, Borghi A, Toni G, Minghetti S, Corazza M. (2014) First randomized trial on clobetasol propionate and mometasonefuroate in the treatment of vulvar lichen sclerosus: results of efficacy and tolerability. Br J Dermatol. 171(2): 38896.

13. Tadir Y, Gaspar A, Lev-Sagie A, Alexiades M, Alinsod R, Bader A, et al. (2017) Light and energy based therapeutics for genitourinary syndrome of menopause: Consensus and controversies. Lasers Surg Med. 49(2): 137-159. doi: 10.1002/1sm.22637. Epub 2017 Feb 21. 